\title{
Doppler Ambiguity Resolution Based on Random Sparse Probing Pulses
}

\author{
Yunjian Zhang, ${ }^{1}$ Zhenmiao Deng, ${ }^{1}$ Jianghong Shi, ${ }^{1}$ Linmei Ye, \\ Maozhong Fu, ${ }^{1}$ and Chen Zhao ${ }^{2}$ \\ ${ }^{1}$ School of Information Science and Engineering, Xiamen University, Fujian 361005, China \\ ${ }^{2}$ Faculty of Science, University of Auckland, Auckland 1052, New Zealand
}

Correspondence should be addressed to Zhenmiao Deng; dzm_ddb@xmu.edu.cn

Received 20 January 2015; Accepted 27 April 2015

Academic Editor: Igor Djurović

Copyright (C) 2015 Yunjian Zhang et al. This is an open access article distributed under the Creative Commons Attribution License, which permits unrestricted use, distribution, and reproduction in any medium, provided the original work is properly cited.

A novel method for solving Doppler ambiguous problem based on compressed sensing (CS) theory is proposed in this paper. A pulse train with the random and sparse transmitting time is transmitted. The received signals after matched filtering can be viewed as randomly sparse sampling from the traditional fixed-pulse repetition frequency (PRF) echo signals. The whole target echo could be reconstructed via CS recovery algorithms. Through refining the sensing matrix, which is equivalent to increase the sampling frequency of target characteristic, the Doppler unambiguous range is enlarged. In particular, Complex Approximate Message Passing (CAMP) algorithm is developed to estimate the unambiguity Doppler frequency. Cramer-Rao lower bound expressions are derived for the frequency. Numerical simulations validate the effectiveness of the proposed method. Finally, compared with traditional methods, the proposed method only requires transmitting a few sparse probing pulses to achieve a larger Doppler frequency unambiguous range and can also reduce the consumption of the radar time resources.

\section{Introduction}

Long range radars (LRRs) usually work in low Pulse Repetition Frequency (PRF). Although there is no or low range ambiguity, it suffers from serious Doppler or velocity ambiguity. Therefore, Doppler ambiguity resolution is necessarily required to ensure accurate measurement of the radial velocity of a target. One resolution introduced from pulse Doppler technology is adopted by LRRs, which measure the velocity by transmitting high PRF impulse train. However, the high PRF mode requires more radar time resources, which are very precious to phased array radars when tracking multiple targets at the same time. On the other hand, the low signal pulse width may lead to a weak corresponding echo signal and hence a small operational range of the LRR, due to the high repetition frequency.

Many Doppler ambiguity resolution methods have been proposed in [1-7]. The so-called robust refinement Chinese Remainder Theorem (CRT) algorithm under noisy environment is proposed in [1-4]. The staggered PRF is proposed in [5] for overcoming Doppler ambiguity. Clearly, by exploiting the difference of the measured Doppler frequencies between two staggered PRF pulses, the blind frequency could be expanded by ten times or more [5]. In [6], a CS-based Doppler ambiguity resolution method is proposed, which can resolve Doppler ambiguities of several targets. The clutter suppression and velocity resolution in moving target indication are investigated in [7].

In this paper, we proposed a novel method to solve Doppler ambiguous problem, which tremendously increases fixed-PRF and randomly determines the small amount of measurement corresponding to the time sequence of transmitted probing pulses. Explicitly, a sparse pulse train is randomly selected from traditional fixed repetition frequency pulses, according to the Restricted Isometry Property (RIP) of the CS sensing matrix, and a random perturbation item is added to the transmitting time of each selected pulse before transmitting. The received signals after Matched Filtering (MF) can be considered as randomly 
sparse sampling of Doppler. By using the CS recovery algorithms, the sensing matrix can be built based on the transmitting time sequence and recover the whole Doppler spectrum. As a result, the number of columns of sensing matrix becomes quite large such that many reconstruction algorithms of CS are not adequate, namely, $l_{1}$-minimization [8], Orthogonal Matching Pursuit (OMP) [9], Spectral Compressive Sensing (SCS) [10], and so forth, since these algorithms require explicit operations on the complete sensing matrix, which introduces extremely high computational complexity, especially in the scenario of large-scale applications.

To address this problem, the proposed method for enlarging the Doppler unambiguous range in this paper is based on Complex Approximate Message Passing (CAMP) algorithm [11], which is an extension of the original Approximate Message Passing (AMP) algorithm [12] from real number field to complex domain. Moreover, the process of message passing algorithm only involves the sensing matrix or transposition of the sensing matrix, and hence, it is especially suitable for radar applications in which the echo signals are often complex [13] and the range of Doppler frequencies for unknown targets is large.

The performance of the CAMP algorithm can be accurately predicted by State Evolution (SE) formalism introduced in [12]. In this formalism, the Mean Square Error (MSE) of reconstruction is a state variable; its change from iteration to iteration is modeled by a scalar function. Therefore, the variance caused by each iteration can be obtained accurately. Combining CRLB results of frequency estimation with nonuniform sampling, the CRLB of the proposed method can be derived.

The rest of the paper is organized as follows. In Section 2, we present the radar echo signal model utilized for this work and the brief revision of CS theory. Section 3 discusses the proposed Doppler ambiguity resolution method based on the adaptive CAMP algorithm in detail. In Section 4, sensing matrix design and radar dwell scheduling scheme are explained. We also derive the Cramer-Rao Lower Bound (CRLB) for the proposed method. We then present numerical simulation results to demonstrate the improvement of the proposed method in Section 5. Finally, we conclude the paper in Section 6.

\section{Model and the CS Theory}

2.1. Radar Echo Signal Model. Let the center frequency to be denoted by $f_{c}$ and the chirp rate to be denoted by $\gamma$; the transmitted chirp signal can be modeled as

$$
s\left(\widehat{t}, t_{m}\right)=\operatorname{rect}\left(\frac{\widehat{t}}{T_{p}}\right) \exp \left(j 2 \pi\left(f_{c} t+\frac{1}{2} \gamma \widehat{t}^{2}\right)\right),
$$

where $\operatorname{rect}(u)=\left\{\begin{array}{l}1,|u| \leq 0.5 \\ 0,|u|>0.5\end{array}\right.$ and $T_{p}$ and $\widehat{t}$ are the pulse width and the fast time, respectively; $t=\widehat{t}+t_{m}$ is the full time, where $t_{m}$ denotes the slow time. When the distance between the target and the radar is $R_{t}$, the received echo signal $s_{r}\left(t, t_{m}\right)$ can be defined as

$$
\begin{aligned}
s_{r}\left(\widehat{t}, t_{m}\right)= & \operatorname{Arect}\left(\frac{\left(\hat{t}-2 R_{t} / c\right)}{T_{p}}\right) \\
& \cdot \exp \left(j \pi \gamma\left(\hat{t}-\frac{2 R_{t}}{c}\right)^{2}\right) \exp \left(-\frac{j 4 \pi f_{c}}{c R_{t}}\right),
\end{aligned}
$$

where $A$ is the coefficient scattering and $c$ is the propagation speed of electromagnetic wave. Performing MF to the received signal and transforming the results into rangefrequency domain yield

$$
r\left(f_{r}, t_{m}\right)=A^{\prime} \operatorname{rect}\left(\frac{f_{r}}{B}\right) \exp \left(-\frac{j 4 \pi\left(f_{r}+f_{c}\right) R_{t}}{c}\right),
$$

where $B$ is the signal bandwidth. Here, $R_{t}$ could be approximated by ignoring the acceleration, jerk, and rotation velocity of the object, leading to

$$
R_{t} \approx R_{0}+v t_{m}
$$

where $R_{0}$ is the distance between the target and radar at time $t_{0}$ and $v$ is the radial velocity. By substituting (4) into (3), the slow-time domain echo signal is obtained as

$$
r\left(f_{d}, t_{m}\right)=A^{\prime \prime} \exp \left(-j 2 \pi f_{d} t_{m}\right)
$$

where $f_{d}=2\left(f_{r}+f_{c}\right) v / c, A^{\prime \prime}=A^{\prime} \exp \left(2\left(f_{r}+f_{c}\right) R_{0} / c\right)$, and $f_{d}$ is the Doppler frequency.

As can be seen from (5), the slow-time domain radar echo signal could be viewed as a sinusoidal signal, which is sparse in frequency domain. Therefore the Doppler frequency estimation problem could be solved via CS theory. Furthermore, if there are $K$ targets with different Doppler frequencies, the echo signal can be rewritten as

$$
r\left(f_{d_{i}}, t_{m}\right)=\sum_{i=1}^{K} A_{i}^{\prime \prime} \exp \left(-j 2 \pi f_{d_{i}} t_{m}\right),
$$

where $f_{d_{i}}$ notes the $i$ th Doppler frequency.

2.2. Compressed Sensing Theory. The CS theory is able to reconstruct the original signal from a set of nonadaptive measurements sampled at a much lower rate than required by the Nyquist sampling theorem by simultaneously sensing and compressing sparse or compressible signals. The original signal denoted by $x \in C^{N}$ and the sampled linear measurements $y \in C^{M}$ can be written as

$$
y=A x+n
$$

where $A \in C^{M \times N}$ denotes the sensing matrix with $M<N$ and $n \in C^{M}$ is the measurement noise. Note that any signal $x$ can be represented in terms of a basis of $N \times 1$ vectors $\left\{\psi_{i}\right\}_{i=1}^{N}$. According to the $N \times N$ basis matrix $\Psi=\left[\psi_{1}\left|\psi_{2}\right|, \ldots, \psi_{N}\right]$ with the vectors $\left\{\psi_{i}\right\}$ as columns, the original signal $x$ can be expressed as $x=\sum_{i=1}^{N} s_{i} \psi_{i}=\Psi s$ where $s_{i}$ represents 


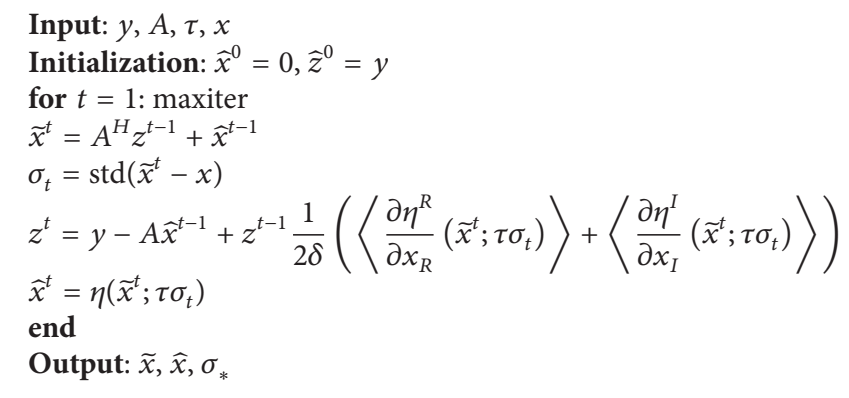

Algorithm 1: Ideal CAMP algorithm.

weighting coefficient. If $x$ is sparse or compressible, and $\Psi$ is chosen properly, the vector $s$ can be computed directly from $y$ provided that the Restricted Isometry Property (RIP) condition holds. The undersampling ratio, that is, the aspect ratio of the sensing matrix, is defined as $\delta \triangleq M / N$ and the sparsity rate is defined as $\rho \triangleq k / M$, where $k$ is the number of nonzero entries. By using the convex optimization, a close approximation of $x$ is expressed as

$$
\widehat{x}=\arg \min _{x^{\prime}}\left\|x^{\prime}\right\|_{1} \text { s.t. }\left\|A x^{\prime}-y\right\|_{2}^{2} \leq \varepsilon
$$

where $\varepsilon$ indicates the noise level. However, in some interesting large-scale applications, since the matrix $A$ and vector $x$ may contain millions of entries, it may become significantly slow to solve the standard convex optimization problem as the Doppler ambiguity problem we addressed previously. Furthermore, the original signal needed to be reconstructed contains enormous number of entries, due to the fact that the practical Doppler frequency of target may be much higher than the PRF of radar. Therefore, it becomes the interest of CS research community to solve the $l_{1}$-penalized least squares problem, expressed as

$$
\widehat{x}=\min _{x^{\prime}} \frac{1}{2}\left\|y-A x^{\prime}\right\|_{2}^{2}+\lambda\left\|x^{\prime}\right\|_{1},
$$

where $\lambda$ is a positive regularization parameter, which represents a tradeoff between sparsity and reconstruction error; that is greater $\lambda$ denotes greater sparsity and greater reconstruction error and vice versa. The complex $l_{1}$-norm is defined as $\|x\|_{1} \triangleq \sum_{i}\left|x_{i}\right|=\sum_{i} \sqrt{\left(x_{i}^{R}\right)^{2}+\left(x_{i}^{I}\right)^{2}}$. Note that this is also referred to as Complex Least Absolute Shrinkage and Selection Operator (c-LASSO) problem [14] in other research fields.

\section{The CAMP Algorithm}

3.1. Adaptive CAMP Algorithm. The CAMP is a fast and efficient iterative algorithm for solving the problem with complex vectors and matrices involved. In the real number field, the algorithm is referred to as AMP algorithm, which only requires the transpose operation on the sensing matrix $A$ [12]. Therefore, it indicates that the analogous method can be applied in complex domain, namely, replacing the matrix $A^{T}$ by $A^{H}$, where $H$ denotes the complex conjugate transpose. As reported in [11], the performance of CAMP has been asymptotically analyzed in the case of noise-free and noisy measurements; here the brief revision of the basics of CAMP is discussed as follows.

In the ideal scenario, the complex original signal $x$ that we intend to reconstruct is assumed to be known at the receiver; thus the exact current standard deviation of the noise is available during the iterative processing. In this paper, this is referred to as Algorithm 1.

Clearly, in Algorithm 1, the complex soft thresholding is the function of $u$ and $\lambda$, as expressed as $\eta(u ; \lambda) \triangleq(|u|-$ $\lambda) e^{j \measuredangle u} \mathbf{1}(|u|>\lambda)$, where $\mathbf{1}$ is the so-called indicator function. The main purpose of the thresholding function is to impose the sparsity at each iteration. $\partial \eta^{R} / \partial x_{R}$ is the partial derivative of $\eta^{R}$ with respect to the real part of the input and $\partial \eta^{I} / \partial x_{I}$ is the partial derivative of $\eta^{I}$ with respect to the imaginary part of the input. The predefined maximum number of iterations is named maxiter. The estimation of $x$ at iteration $t$ is labeled as $\widehat{x}^{t}$, and $\tilde{x}^{t}$ is a nonsparse, noisy estimate of $x$, where $\sigma_{t}$ is the standard deviation and $\langle\cdot\rangle$ denotes arithmetic average.

Algorithm 1 first searches for a noisy estimation of the original signal $\tilde{x}^{t}$, which is not sparse. In order to obtain the sparse estimation $\widehat{x}^{t}$, the soft thresholding function is subsequently adapted. Here the assumption is made that the algorithm uses the known $x$ for calculating the noise standard deviation $\sigma_{t}$. However, in fact, the original signal $x$ we intend to reconstruct is unknown. An alternative approach of estimating $\sigma_{t}$ is adopted to tackle it, which utilizes median estimation shown as

$$
\widehat{\sigma}_{t}=\sqrt{\frac{1}{\ln 2}} \operatorname{median}\left(\left|\tilde{x}^{t}\right|\right) .
$$

Furthermore, the optimal CAMP thresholding control parameter $\widehat{\tau}_{o}$ (typically in the range $2<\tau_{o}<4$ [15]) can be derived based on the estimation of $\sigma_{t}$, which minimizes $\widehat{\sigma}_{*}^{2}$. Note that it is assumed that we know $\tau_{\max }$ such that $\tau_{o}<\tau_{\max }$. Given a step $\delta_{\tau}$, we define a sequence of thresholds $\tau=\left\{\tau_{l}\right\}_{l=1}^{L}$ such that $\tau_{1}=\tau_{\max }$ and $\tau_{l}=\tau_{l-1}-\delta_{\tau}$. Starting from $\tau_{\max }$, at the first iteration $l$ CAMP is initialized with $\widehat{x}_{l}^{0}=\widehat{x}_{l-1}$ and $z_{l}^{0}=z_{l-1}$. Using the solution of CAMP at the previous iteration $l-1$ as initial value for $\tau=\tau_{l}$. 


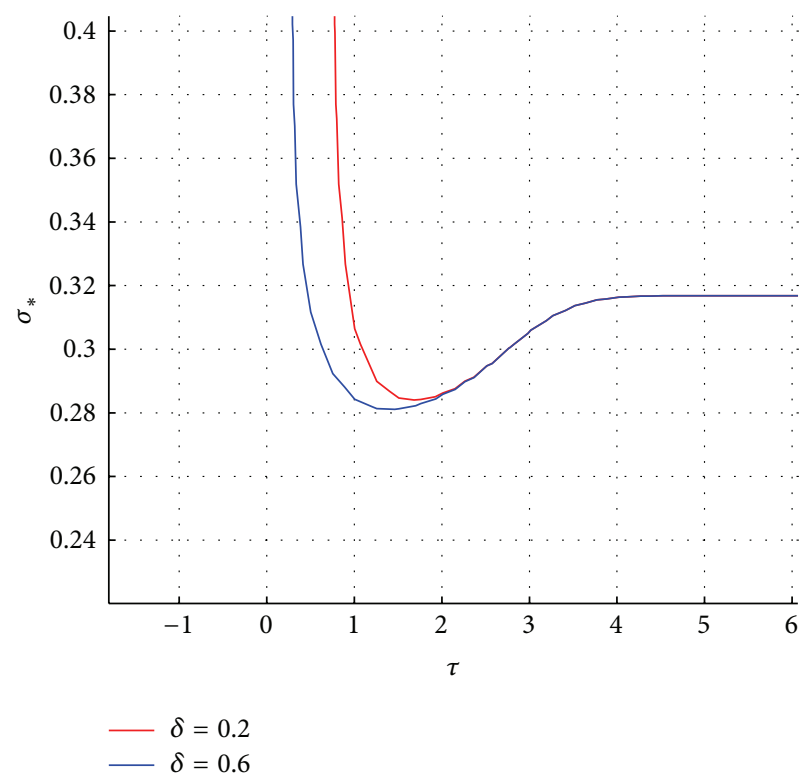

(a) $\delta=0.2$ and 0.6

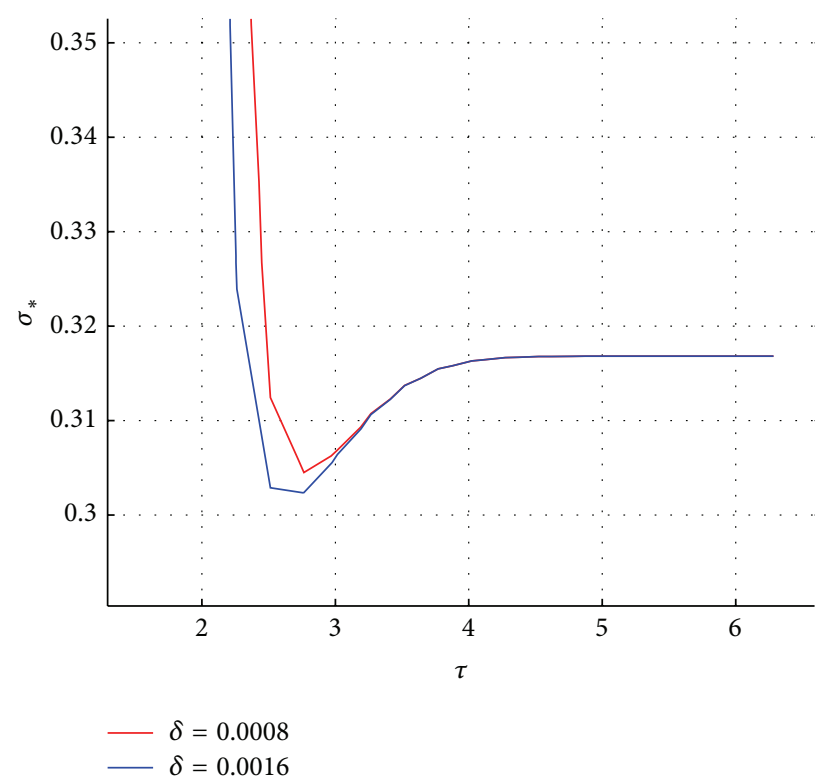

(b) $\delta=0.0008$ and 0.0016

Figure 1: Fixed point $\sigma_{*}$ versus optimal threshold $\tau_{o}$ for ideal CAMP with $\sigma=0.23$; undersampling ratios $\delta$ equal (a) $0.2,0.6$ and (b) 0.0008 , 0.0016 , respectively. Sparsity rate $\rho=0.1$ and the nonzero entries of vector $x$ have all amplitudes equal to 1 and phase uniformly distributed between $-\pi$ and $\pi$.

After $L$ iterations, the resultant matrix $\widehat{X}=\left[\widehat{x}_{1}, \widehat{x}_{2}, \ldots, \widehat{x}_{L}\right]$ of size $N \times L$ contains the CAMP solution for a given $\tau_{l}$ in each column. Also, we have $L$ estimates $\left\{\widehat{\sigma}_{*}^{l}\right\}_{l=1}^{L}$. The optimal threshold $\widehat{\tau}_{o}$ is chosen as the one that minimizes the estimated CAMP output noise variance $\widehat{\sigma}_{*}^{2}$. The value of $\tau_{\max }$ can be determined with assist of the c-LASSO problem. It is known that for $\lambda>\lambda_{\max }=\left\|A^{H} y\right\|_{\infty}$ the only solution is zero solution. One uses the calibration equation [11], which denotes the relationship between $\lambda$ and $\tau$, to evaluate the $\widehat{\tau}_{\max }$. The calibration equation is given by

$$
\begin{aligned}
\lambda \triangleq & \approx \sigma_{*}\left(1-\frac{1}{2 \delta} E\left(\frac{\partial \eta^{R}}{\partial x_{R}}\left(X+\sigma_{*} Z ; \tau \sigma_{*}\right)\right.\right. \\
& \left.\left.+\frac{\partial \eta^{I}}{\partial x_{I}}\left(X+\sigma_{*} Z ; \tau \sigma_{*}\right)\right)\right),
\end{aligned}
$$

where $E$ is with respect to the two independent random variables $Z \sim C N(0,1)$ and $X \sim p_{X}$. With $\lambda=\lambda_{\max }$ and $\sigma_{*}=\widehat{\sigma}_{0}$, the value of the estimation $\widehat{\tau}_{\max }$ can be evaluated. The expectation in (11) equals the MSE of the estimate $\hat{x}^{t}$ after applying the soft thresholding function. The details in evaluating the function with a given $p_{X}$ are shown in [13, Appendix A]. This algorithm is called Adaptive CAMP, since both the noise variance $\widehat{\sigma}_{t}$ and the threshold $\widehat{\tau}_{o}$ are adaptively estimated inside the algorithm itself, and the only input variables are $y$ and $A$. The relationship of fixed-point $\sigma_{*}$ and the optimal threshold $\tau_{o}$ can be expressed as

$$
\sigma_{t+1}^{2}=\sigma^{2}+\frac{1}{\delta} E\left(\left|\eta\left(X+\sigma_{t} Z ; \tau \sigma_{t}\right)-X\right|^{2}\right)
$$

by using SE formalism. Figure 1 demonstrates the relation when different values are chosen for the undersampling ratios. Equation (12) exhibits that the time $t+1$ deviation is constituted by the input noise variance $\sigma^{2}$ and the MSE of the estimation after applying the soft thresholding function. Then we can analytically calculate the time $t+1$ deviation caused by CAMP. It has been proved that the right-hand side of (12) is concave [11]; thus there exists at most one stable fixed point $\sigma_{*}^{2}$. Moreover, for $\tau=\tau_{o}$, the value of $\sigma_{*}$ for DFT frame is not very different from the value for Gaussian matrix. For extremely sparse situation where the number of Doppler frequencies is much lower than the number of frequency points, for example, the Doppler ambiguity problem, the relation between $\sigma_{*}$ and $\tau_{o}$ is shown in Figure 1(b). Furthermore, from Figure 1 we can find that both the optimal threshold $\tau_{o}$ and the corresponding output noise standard deviation increase as the undersampling ratio $\delta$ decreases; namely, the number of measurements decreases.

\section{CS Sensing Matrix Design and Radar Dwell Scheduling}

4.1. Sensing Matrix Design. To construct the measurement matrix $\Phi$, the $M$ number of rows form an $N \times N$ identity matrix is selected, which corresponds to the time sequence of transmitted probing pulses. In order to increase unambiguous Doppler range, $M$ pulses from the traditional fixed-PRF pulse train are selected randomly and a random perturbation is added to the transmitting time of every selected pulse.

Since $r\left(f_{d}, t_{m}\right)$ in (5) is sparse in frequency domain, the basis matrix $\Psi$ can be defined as Discrete Fourier Transform (DFT) of $x$ :

$$
\Psi_{i, k}=\exp \left(-\frac{j 2 \pi i k}{N}\right), \quad 0 \leq i, k \leq N .
$$


The sensing matrix $A$, also known as "randomly extracting matrix," is evaluated by the measurements matrix $\Phi$ multiplying the basis matrix $\Psi$. Note that the sensing matrix $A$ is equivalent to an oversampled DFT matrix, that is, DFT frame.

4.2. Radar Dwell Scheduling. The PRF of LRR is usually low and the typical value of $f$ is $10 \mathrm{~Hz}<f<1000 \mathrm{~Hz}$. When radar works in fixed PRF mode, the pulse transmitting time sequences are $t_{i}=i / f_{s}, i=1, \ldots, n$. In this case, the echoes in the slow-time domain may suffer from severe Doppler ambiguity. By modifying $t_{i}$ as $t_{i}^{\prime}=t_{i}+\Delta_{i}$, where $\Delta_{i}$ is a random perturbation, we can obtain a new transmitting time sequence, which is equivalent to those extracted from a high PRF pulse transmitting time set. It is obvious that the precision of perturbation determines the equivalent value of PRF. The slow-time domain echo signal could be recovered by CAMP algorithms from the measurements $y$ as discussed before.

Clearly, $M$ elements are randomly selected from the transmitting time set $\left\{t_{1}, t_{2}, \ldots, t_{n}\right\}$ and each of them is added by a perturbation $\Delta_{k}$, respectively, where $\Delta_{k}$ is a random variable with its mean and variance to be 0 and $\xi^{2}$, labeled as $\Delta_{k} \sim N\left(0, \xi^{2}\right)$. The pulse transmitting time can be expressed as

$$
t_{k}^{\prime}=t_{k}+\Delta_{k}
$$

where $k$ is the time index and is a subset of $\{1,2, \ldots n\}$; that is, $k=\left\{k_{1}, k_{2}, \ldots, k_{M}\right\} \subset\{1,2, \ldots, n\}$.

In practical, the term $\Delta_{k}$ is not completely random, which is caused by the factor that the time sequence of a radar controlled by a reference clock, which indicates that the precision of $\Delta_{k}$ should be lower than that of clock. Actually, high precision of $\Delta_{k}$ means large unambiguous Doppler range; meanwhile, the refining factor is enlarged, which introduces high computational burden. Therefore, to ensure enough Doppler unambiguous range, a proper value of $\Delta_{k}$ is necessarily required. Here, assuming the time precision is $\Delta t$, the corresponding equivalent $\mathrm{PRF}$ is $f_{s}^{\prime}=1 / \Delta t$, and the Doppler unambiguous range for $t_{i}^{\prime}$ is represented as

$$
f_{s}^{\prime}=\frac{1}{\Delta t} \cdot f_{s}
$$

The unambiguous Doppler range is extended to $f_{s}^{\prime}$. The purpose of enlarging unambiguous Doppler range is to widen the measure velocity range, which is equal to

$$
v=\frac{c f_{s}^{\prime}}{2\left(f_{c}+f_{r}\right)}=\frac{c f_{s}^{\prime}}{2\left(f_{c}+\gamma t_{m}\right)} .
$$

It is obvious that the velocity of resolution $\Delta v$ depends on precision of $f_{s}^{\prime}$, according to (16).

After determining the dwell time $t_{1}, t_{2}, \ldots, t_{M}$, radar transmits pulses at these moments. The measurements $r_{1}, r_{2}, \ldots, r_{M}$ in slow-time domain are obtained by matched filtering to the echo signals. By using the CAMP algorithm, the slow-time domain echo signals can be recovered and the Doppler frequency under expanded unambiguous Doppler range can be estimated. The estimation accuracy is determined by the precision of the basis matrix. In order to obtain more accurate Doppler estimate, an adaptive refinement algorithm of the sensing matrix proposed in [16] can be used to reconstruct the slow-time domain signal with higher precision.

4.3. SNR and CRLB. The definition of SNR for the CAMPbased CS radar system is provided by [13, Ch. 3.4]. The SNR at the input $\left(\mathrm{SNR}_{\mathrm{in}}\right)$ and output $(\mathrm{SNR})$ of the $\mathrm{MF}$ are defined by

$$
\begin{aligned}
\mathrm{SNR}_{\mathrm{in}, \mathrm{MF}} & =\frac{a^{2}}{N \sigma^{2}}, \\
\mathrm{SNR}_{\mathrm{in}, \mathrm{CAMP}} & =\frac{a^{2}}{M \sigma^{2}}, \\
\mathrm{SNR}_{\mathrm{MF}} & =\frac{a^{2}}{\sigma^{2}}, \\
\mathrm{SNR}_{\mathrm{CAMP}} & =\frac{a^{2}}{\sigma_{*}^{2}},
\end{aligned}
$$

where $a^{2}$ is the received power of a target at bin $i$ and $\sigma_{*}$ is the minimized standard deviation defined in Section 3. Based on previous analysis, the $\mathrm{SNR}_{\mathrm{CAMP}}$ is lower than $\mathrm{SNR}_{\mathrm{MF}}$ due to the increase of noise standard deviation imposed by the iterative CAMP algorithm.

Consider the case of $M$ samplings of a single complex sinusoid corrupted by Additive White Gaussian Noise (AWGN):

$$
\begin{aligned}
x_{t_{m}} & =A e^{j\left(\omega t_{m}+\theta\right)}+n_{t_{m}}, \\
\mathbf{x} & =\left[x_{t_{0}}, x_{t_{1}}, \ldots, x_{t_{M-1}}\right]^{T},
\end{aligned}
$$

where $\left\{t_{m} \mid 0 \leq m \leq M-1\right\}$ is an index set for the sampling time, $\omega$ is the unknown frequency in radians, $\theta$ is a random phase which is uniformly distributed over $[-\pi, \pi], n_{t}$ is zero mean complex AWGN with variance $\sigma^{2}$, and $A$ is the energy per sampling.

The conditional Probability Density Function (PDF) of $\mathbf{x}$ given $\omega$ and $\theta$ is

$$
\begin{aligned}
p(\mathbf{x} \mid \omega, \theta)= & \frac{\exp \left\{-\left(\|\mathbf{x}\|^{2}+M A\right) / \sigma^{2}\right\}}{\left(\pi \sigma^{2}\right)^{M}} \\
& \cdot \exp \left(\frac{2 A}{\sigma^{2}} \operatorname{Re}\left\{\sum_{m=0}^{M-1} x_{t_{m}} e^{-j\left(\omega t_{m}+\theta\right)}\right\}\right) .
\end{aligned}
$$

Taking the log of (19) and neglecting constants yield the log likelihood function for samplings in AWGN

$$
L(\mathbf{x} \mid \omega, \theta)=\frac{2 A}{\sigma^{2}} \operatorname{Re}\left\{\sum_{m=0}^{M-1} x_{t_{m}} e^{-j\left(\omega t_{m}+\theta\right)}\right\}
$$


Fisher's information matrix (FIM) is the expected value of the second order derivatives

$$
\mathbf{J}=\frac{2 A^{2}}{\sigma^{2}}\left[\begin{array}{cc}
\sum_{m=0}^{M-1} t_{m}^{2} & \sum_{m=0}^{M-1} t_{m} \\
\sum_{m=0}^{M-1} t_{m} & M
\end{array}\right] .
$$

Inverting $\mathbf{J}$ yields the CRLB for the estimation variance of the frequency in AWGN. In our proposed method, when the CAMP reconstruction algorithm is applied, the variance $\sigma^{2}$ becomes $\sigma_{*}^{2}$ and the CRLB is given by

$$
\operatorname{CRLB}(\widehat{w})=\frac{1}{A^{2} / \sigma_{*}^{2}} \frac{M / 2}{M \sum_{m=0}^{M-1} t_{m}^{2}-\left(\sum_{m=0}^{M-1} t_{m}\right)^{2}} .
$$

Note that the variance $\sigma_{*}^{2}$ is larger than the variance $\sigma^{2}$, indicating that the CRLB of our proposed method is higher than that of traditional nonuniform sampling methods [17].

\section{Numerical Simulations}

In this section, the performance of proposed method is studied with the aid of numerical simulations. We commence the section by investigating the noise-free scenario, where the unambiguous Doppler frequencies are $345.482 \mathrm{~Hz}$ and $347.158 \mathrm{~Hz}$. Since radar transmits a fixed-PRF $(\mathrm{PRF}=100 \mathrm{~Hz})$ pulse train. It is worth pointing out that the pulse train can be used in some LRRs for the velocity measurement or the normal waveform for tracking targets. $M$ is set to 12 , and the pulse transmitting time set $t_{k}$ is obtained by randomly extracting $M$ elements from the fixed-PRF transmitting time set with the perturbation added. The precision of pulse transmitting time is $\Delta t=0.001$, and the corresponding dimension of basis matrix is $N=1 / \Delta t=1000$. Doppler frequencies are determined corresponding to the peaks of frequency response. The estimated Doppler frequencies are $345 \mathrm{~Hz}$ and $347 \mathrm{~Hz}$, closing to the true values $345.482 \mathrm{~Hz}$ and $347.158 \mathrm{~Hz}$. In order to obtain higher estimate accuracy, redundancy sensing matrix can be refined by multiple. As shown in Figure 2, the Doppler frequencies are estimated as 345.3369 Hz and $347.3057 \mathrm{~Hz}$ with higher accuracy, when the refining factor $q$ is set as 100 , with SNR equal to $20 \mathrm{~dB}$.

Since the tradeoff between success rate of reconstruction and calculation amount is determined by the value of the refining factor, the influence of refining factor for reconstruction performance is investigated as follows. Monte Carlo (MC) simulation results under different refining factors are depicted in Figure 3. The number of iterations is set to 1 and $1000 \mathrm{MC}$ simulations are implemented. The success rate of reconstruction is related to the precision of true frequency $\Delta f$ of the unknown target. As it is clear from Figure 3 as $\Delta f$ increases the curves go up. Higher precision of the frequency of the target requires larger refining factor and vice versa. On the other hand, the significantly low refining factor, namely, the significantly large distance between adjacent columns in the DFT frame, introduces the spectrum leakage, as discussed in $[10]$.

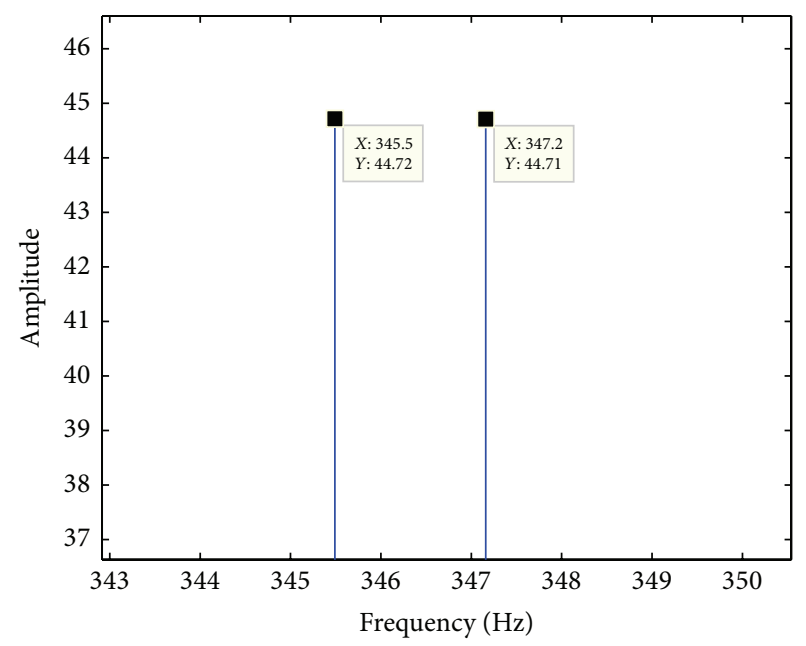

FIGURE 2: Spectrum of recovered slow-time echo signal.

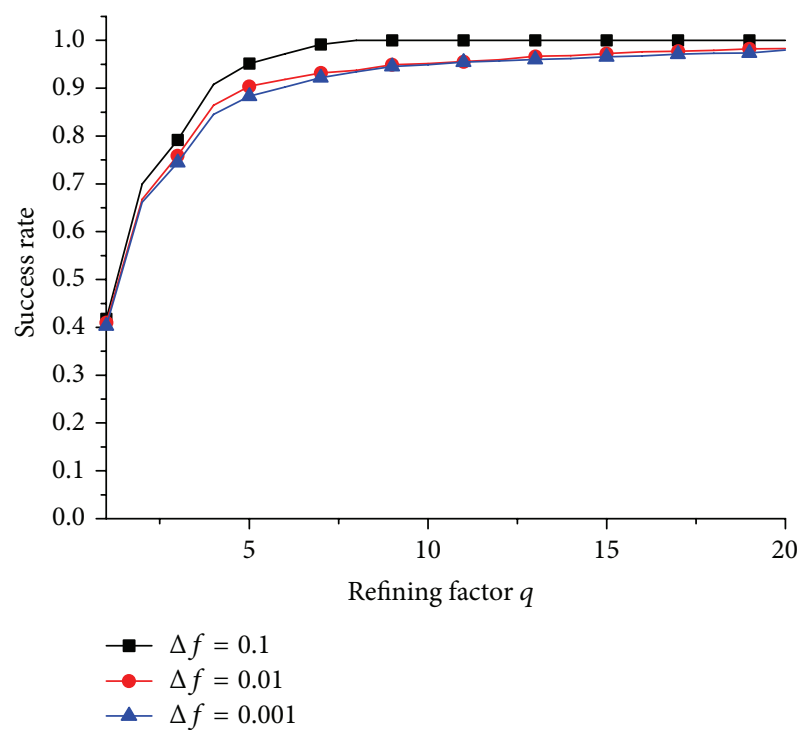

FIGURE 3: The success rate of reconstruction with different refining factor.

TABLE 1: Comparison of simulation times versus different refining factors.

\begin{tabular}{lcccc}
\hline $\begin{array}{l}\text { Refining } \\
\text { factor }\end{array}$ & 10 & 100 & 500 & 1000 \\
\hline Time (s) & 0.044040 & 0.417513 & 2.064184 & 3.909073 \\
\hline
\end{tabular}

The success rate of reconstruction estimated at different SNR is shown in Figure 4, which demonstrates the performance with the presence of noise. Here the number of iterations is set to 31 .

As shown in Figure 4, more than 95\% trials succeed provided that the SNR is greater than $10 \mathrm{~dB}$. Running times under different refining factors are recorded in seconds using the tic/toc functions of Matlab. The frequency span of the sensing matrix is set to $10 \mathrm{kHz}$. Simulation results shown in Table 1 indicate that the proposed method owns good real time response. 
TABLE 2: Compared with CRT, Staggered PRF, and nonuniform sample.

\begin{tabular}{lcccc}
\hline Method & CS & CRT & Staggered frequency & Nonuniform sampling \\
\hline $\begin{array}{l}\text { Sample property } \\
\text { Pulse number }\end{array}$ & $M \ll f_{s}$ & $f_{s 1}+f_{s 2}$ & $f_{s 1}+f_{s 2}$ & $f_{s} \pm \Delta f_{i}$ \\
\hline Unambiguous range & $q \cdot f_{s}$ & $f_{s 1} \cdot f_{s 2}$ & $\left|f_{s 1} \cdot f_{s 2} /\left(f_{s 1}-f_{s 2}\right)\right|$ & $f_{s}$ \\
\hline
\end{tabular}

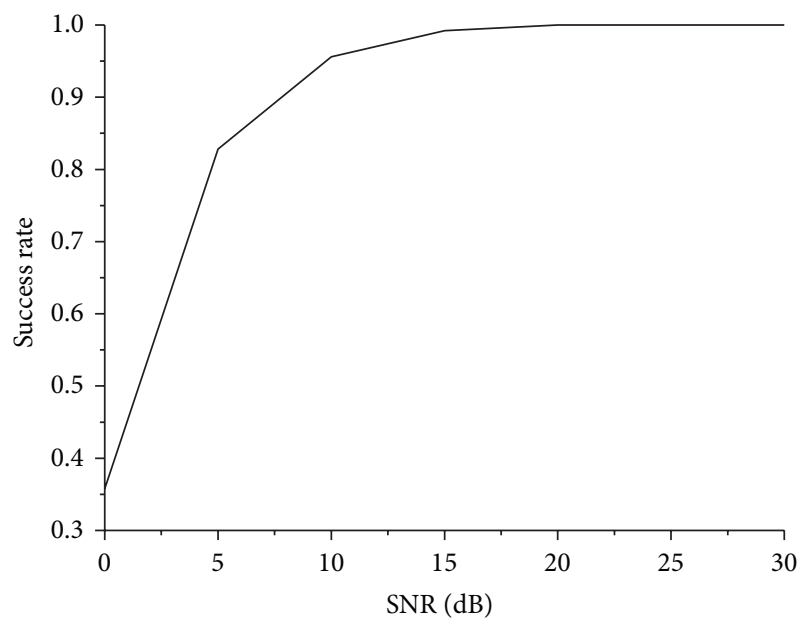

FIGURE 4: The success rate of reconstruction at different SNR.

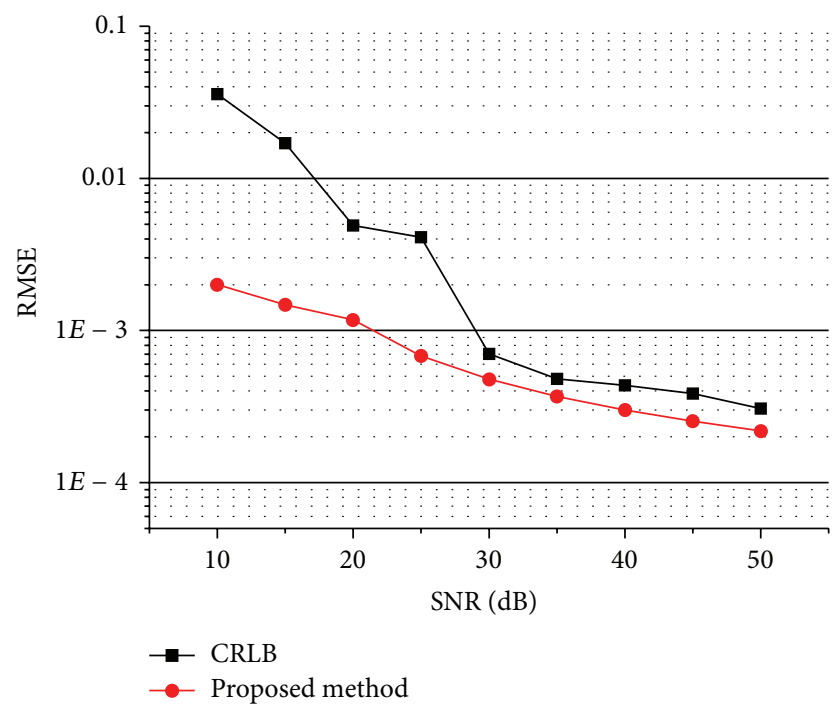

FIGURE 5: Performance of the proposed method.

The comparison between the Root Mean Square Error (RMSE) and the CRLB for frequency estimation of a sinusoid with the presence of noise is investigated, as shown in Figure 5. The refining factor $q$ is set to 1000 and the number of samplings $M$ is set to 12 . The simulation is repeated 1000 times. It is implied from Figure 5 that the Doppler frequency can be obtained from recovered slow-time domain echo signal. The curve of RMSE approaches that of the CRLB due to the error caused by reconstruction which decreases slowly

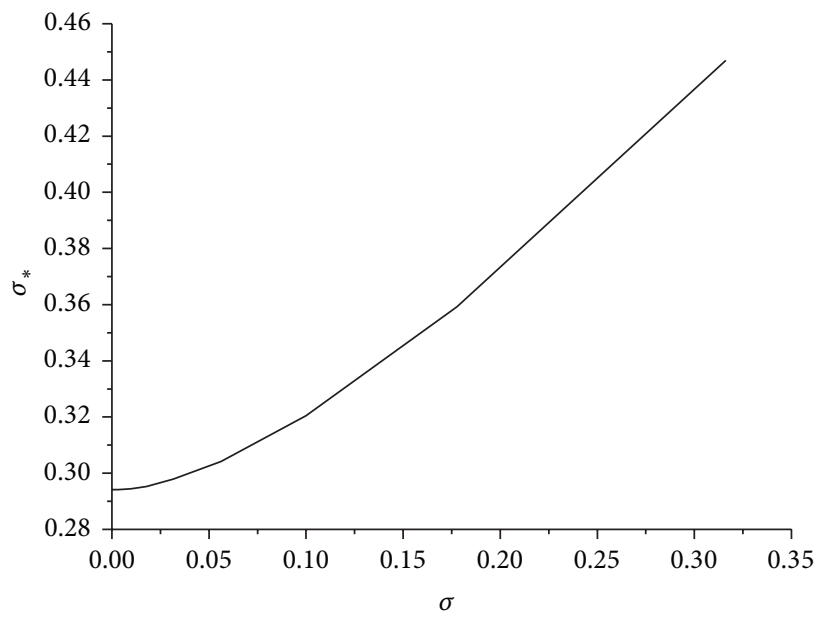

FIGURE 6: The relationship between $\sigma$ and $\sigma_{*}$. The signal amplitude is 1 and the corresponding SNR is set from $10 \mathrm{~dB}$ to $70 \mathrm{~dB}$.

as the SNR increases; thus the SNR defined in (17) increases slowly. Figure 6 demonstrates the relation between $\sigma$ and $\sigma_{*}$, and it is observed that the deviation $\sigma_{*}$ converges to 0.2942 when $\sigma$ decreases to 0 .

In the following, our proposed method is compared with traditional methods, including CRT, Staggered PRF, and nonuniform sample [18], and the comparison results are presented in Table 2. Seen from Table 2, our proposed method transmits the least transmitting pulses. Furthermore, the Doppler frequencies are obtained from recovered Doppler spectrum via CAMP in our proposed method, while unambiguous Doppler frequencies are obtained from ambiguous Doppler measurements in those traditional algorithms.

\section{Conclusion}

Relied on sparse property of radar echo and CS theory, a novel Doppler ambiguous resolution utilizing sparse probing pulses is proposed. The whole slow-time domain echo signals can be recovered via the adaptive CAMP reconstruction algorithm. By refining the basis matrix, the equivalent high PRF echoes can be obtained and therefore, the Doppler unambiguous range can be enlarged. Using the SE formalism, we have derived the CRLB of the proposed method. The accuracy of estimation is demonstrated by theoretical inference and simulations. Compared with traditional methods, our novel method only transmits a few sparse probing pulses and reduces more than $80 \%$ of the time resource consumption of radar. Furthermore, due to the fact that the pulse transmitting 
time can be controlled by the radar clock, which generally has a frequency of $10 \mathrm{MHz}$ or integral multiple of $10 \mathrm{MHz}$, the precision of pulse transmitting time adjustment needed in the paper is achievable. Therefore, our proposed method is adequate to be implemented in practical, since only slight adjustment is required on the radar dwell scheduling.

\section{Conflict of Interests}

The authors declare that there is no conflict of interests regarding the publication of this paper.

\section{Acknowledgments}

The research was supported by the National High-tech R\&D Program of China and the Open-End Fund National Laboratory of Automatic Target Recognition (ATR). The authors would like to thank Laura Anitori and Zai Yang for sharing the CAMP Matlab package.

\section{References}

[1] A. Ferrari, C. Bérenguer, and G. Alengrin, "Doppler ambiguity resolution using multiple PRF," IEEE Transactions on Aerospace and Electronic Systems, vol. 33, no. 3, pp. 738-751, 1997.

[2] X. Li, H. Liang, and X.-G. Xia, "A robust Chinese remainder theorem with its applications in frequency estimation from undersampled waveforms," IEEE Transactions on Signal Processing, vol. 57, no. 11, pp. 4314-4322, 2009.

[3] W.-J. Wang and X.-G. Xia, "A closed-form robust Chinese remainder theorem and its performance analysis," IEEE Transactions on Signal Processing, vol. 58, no. 11, pp. 5655-5666, 2010.

[4] A. Maroosi and H. K. Bizaki, "Digital frequency determination of real waveforms based on multiple sensors with low sampling rates," IEEE Sensors Journal, vol. 12, no. 5, pp. 1483-1495, 2012.

[5] T. Liu and Y.-H. Gong, "Staggered RPF analysis and design," Journal of University of Electronic Science and Technology of China, vol. 38, no. 2, pp. 97-101, 2009.

[6] Y.-X. Zhang, J.-P. Sun, B.-C. Zhang, and W. Hong, "Doppler ambiguity resolution based on compressive sensing theory," Journal of Electronics and Information Technology, vol. 33, no. 9, pp. 2103-2107, 2011 (Chinese).

[7] Y. H. Quan, L. Zhang, M. D. Xing, and Z. Bao, "Velocity ambiguity resolving for moving target indication by compressed sensing," Electronics Letters, vol. 47, no. 22, pp. 1249-1251, 2011.

[8] S. S. Chen, D. L. Donoho, and M. A. Saunders, "Atomic decomposition by basis pursuit," SIAM Journal on Scientific Computing, vol. 20, no. 1, pp. 33-61, 1998.

[9] Y. C. Pati, R. Rezaiifar, and P. S. Krishnaprasad, "Orthogonal matching pursuit: recursive function approximation with applications to wavelet decomposition," in Proceedings of the 27th Asilomar Conference on Signals, Systems \& Computers, pp. 4044, November 1993.

[10] M. F. Duarte and R. G. Baraniuk, "Spectral compressive sensing," Applied and Computational Harmonic Analysis, vol. 35, no. 1, pp. 111-129, 2013.

[11] A. Maleki, L. Anitori, Z. Yang, and R. G. Baraniuk, "Asymptotic analysis of complex LASSO via complex approximate message passing (CAMP)," IEEE Transactions on Information Theory, vol. 59, no. 7, pp. 4290-4308, 2013.
[12] D. L. Donoho, A. Maleki, and A. Montanari, "Message-passing algorithms for compressed sensing," Proceedings of the National Academy of Sciences of the United States of America, vol. 106, no. 45, pp. 18914-18919, 2009.

[13] L. Anitori, Compressive sensing and fast simulations: applications to radar detection [Dissertation], Delft University of Technology, Delft, The Netherlands, 2012.

[14] R. Tibshirani, "Regression shrinkage and selection via the lasso," Journal of the Royal Statistical Society, Series B: Methodological, vol. 58, no. 1, pp. 267-288, 1996.

[15] A. Maleki and D. L. Donoho, "Optimally tuned iterative reconstruction algorithms for compressed sensing," IEEE Journal on Selected Topics in Signal Processing, vol. 4, no. 2, pp. 330-341, 2010.

[16] L. Hu, Z. Shi, J. Zhou, and Q. Fu, "Compressed sensing of complex sinusoids: an approach based on dictionary refinement," IEEE Transactions on Signal Processing, vol. 60, no. 7, pp. 38093822, 2012.

[17] J. A. Gansman, J. V. Krogmeier, and M. P. Fitz, "Single frequency estimation with non-uniform sampling," in Proceedings of the 30th Asilomar Conference on Signals, Systems and Computers, vol. 1, pp. 399-403, IEEE, Pacific Grove, Calif, USA, November 1996.

[18] S.-W. Park, W.-D. Hao, and C. S. Leung, "Reconstruction of uniformly sampled sequence from nonuniformly sampled transient sequence using symmetric extension," IEEE Transactions on Signal Processing, vol. 60, no. 3, pp. 1498-1501, 2012. 

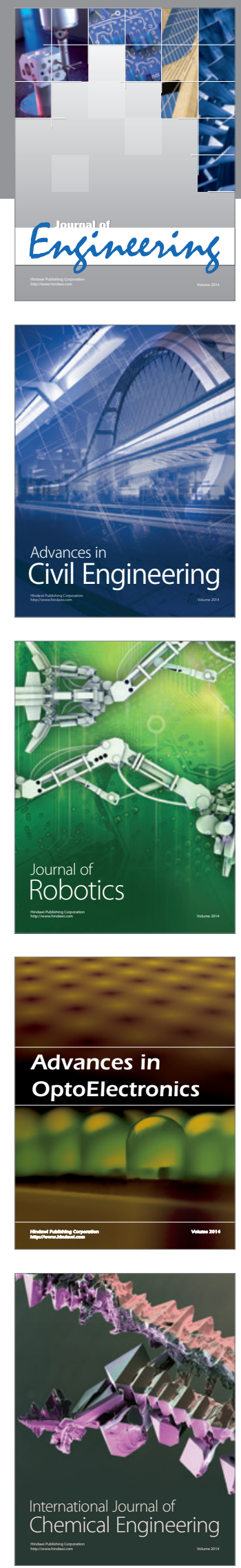

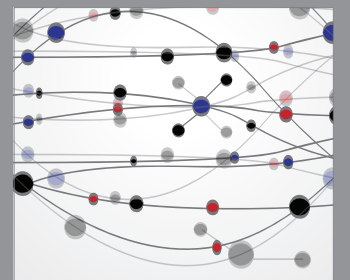

The Scientific World Journal
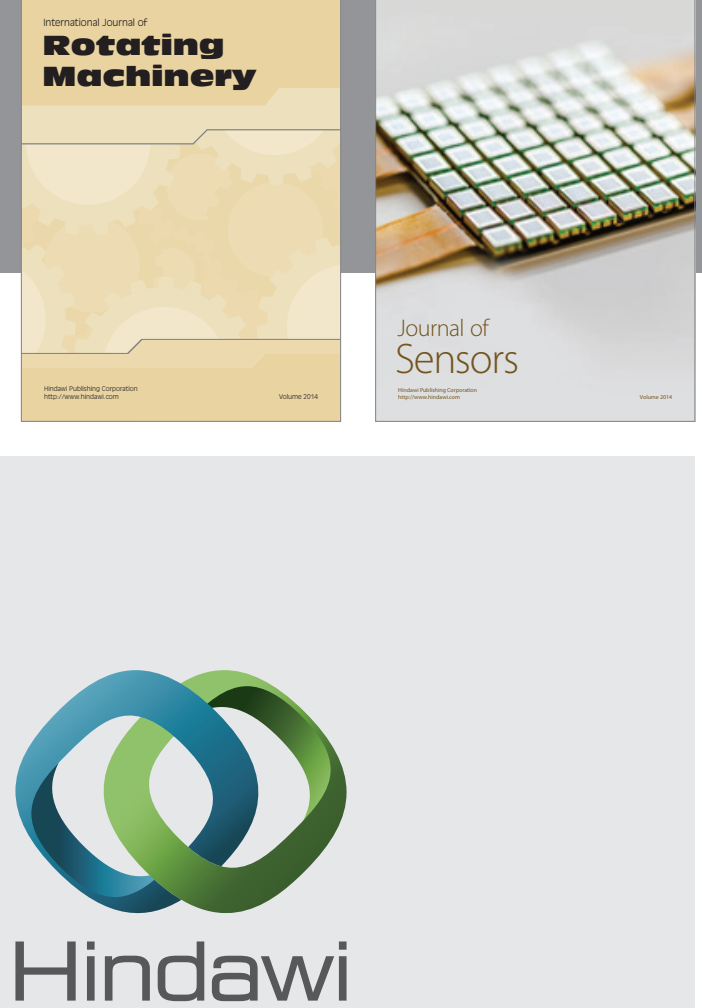

Submit your manuscripts at http://www.hindawi.com
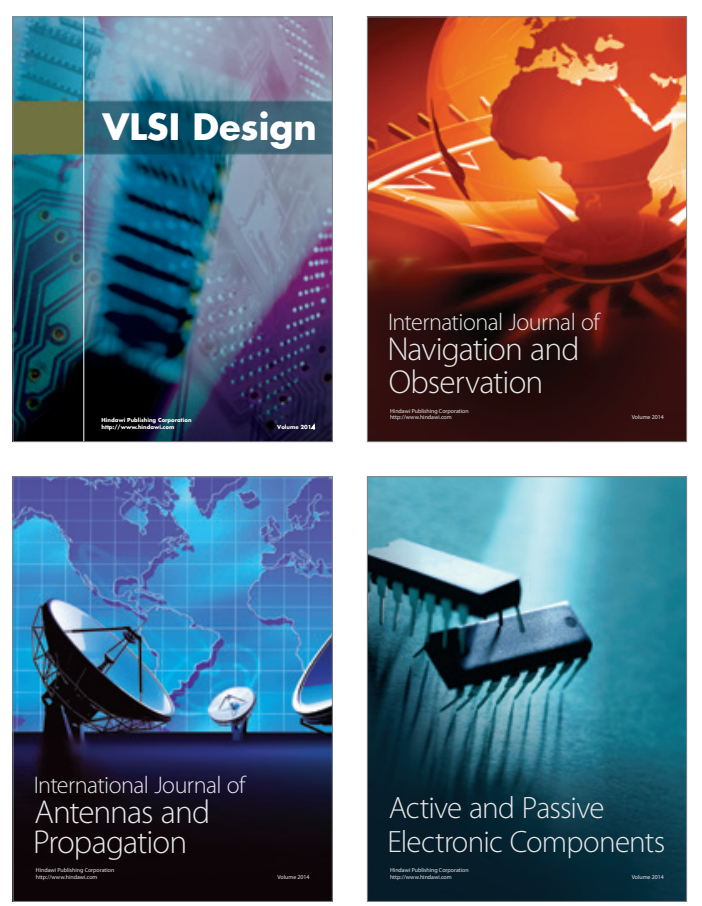
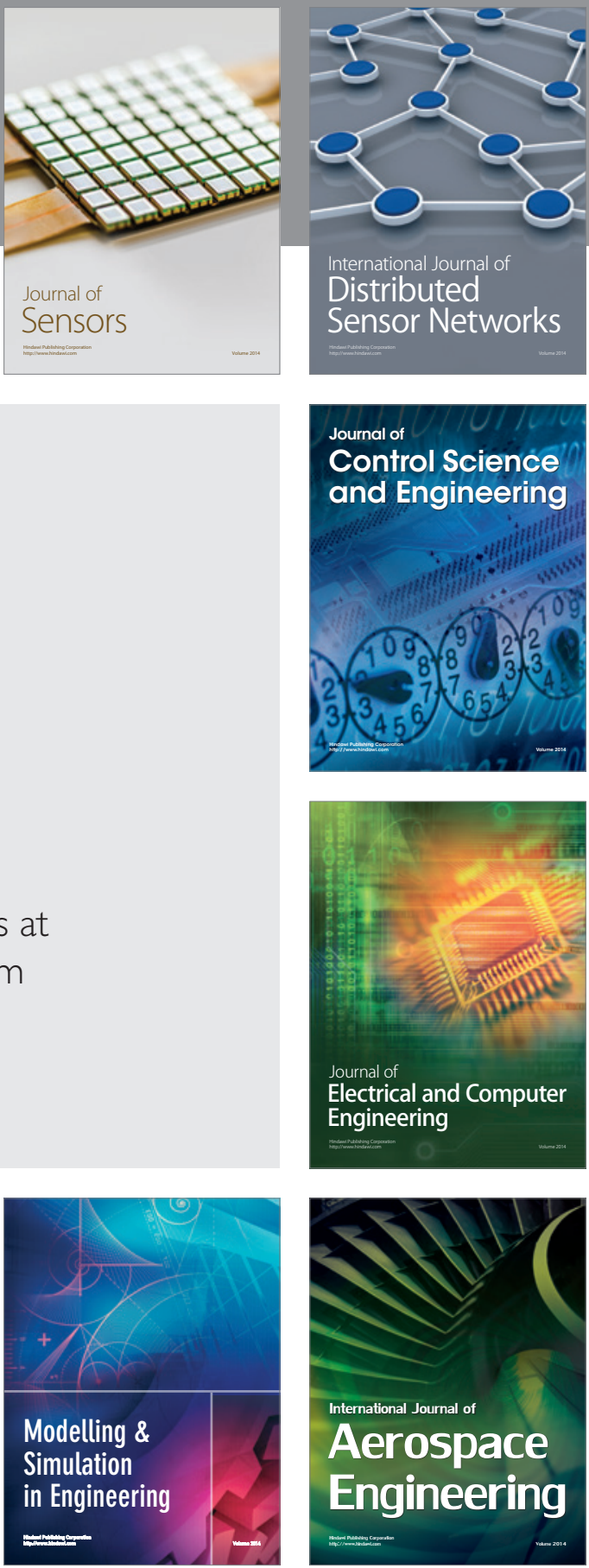

Journal of

Control Science

and Engineering
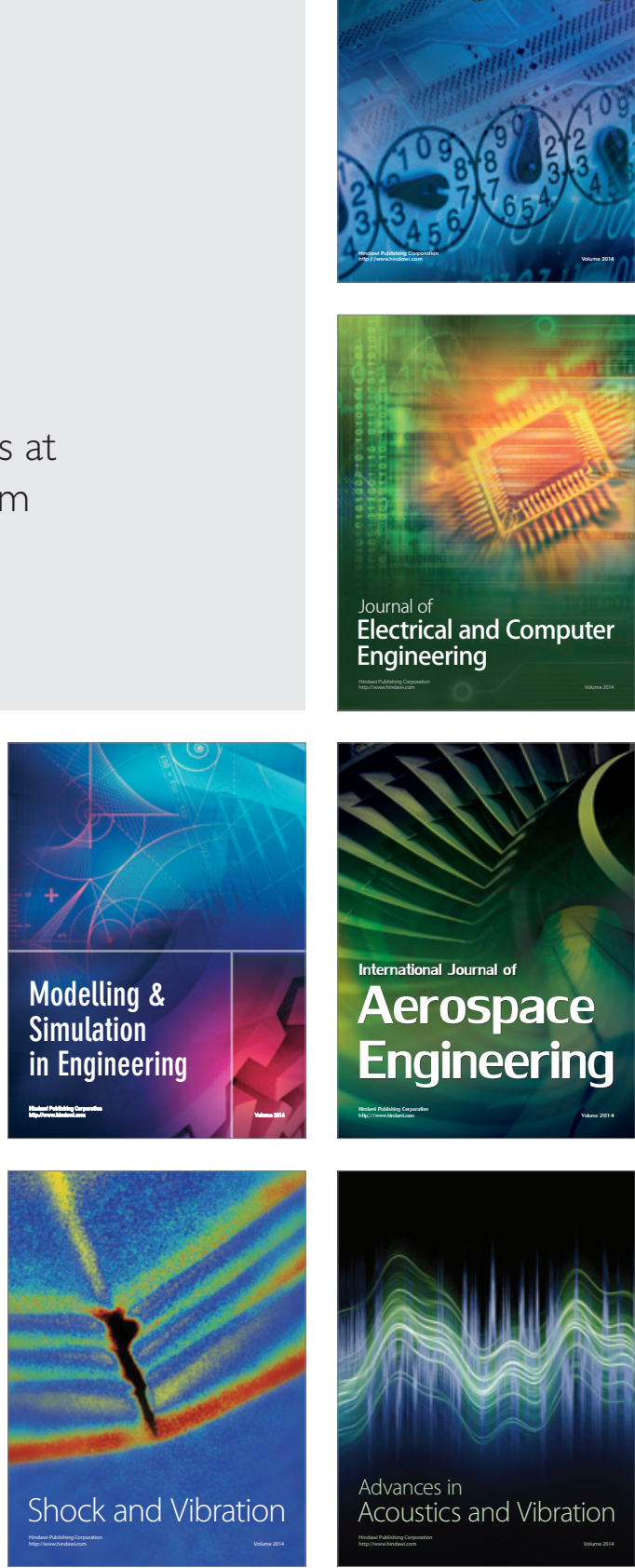